\title{
INDUSTRIA MANUFACTURERA Y CRECIMIENTO ECONÓMICO EN LA PROVINCIA DE TUNGURAHUA
}

\author{
Gladys Gimena Coello Gómez 1
}

Pag 16-18

Fecha de recepción: 5 Diciembre 2016

Fecha de aceptación: 30 Diciembre 2016

\section{Resumen}

a presente investigación, mediante un marco teórico de econoLmistas heterodoxos, con información del Valor Agregado Bruto de 2009 a 2014, pretende contribuir con datos a la evidencia empírica del crecimiento económico de la provincia de Tungurahua, marcado por el desarrollo del sector manufacturero, lo que explica el cumplimiento del principio de causación circular y acumulativo; caracterizado por la interdependencia entre el crecimiento del producto manufacturero y el resto de sectores de la economía de la provincia; lo que a su vez marca la diferencia entre su dinamismo económico y el de las demás provincias de la zona 3 del Ecuador.

Palabras clave: crecimiento económico, manufacturas y causación.

\begin{abstract}
The present research, using a theoretical framework of heterodox economists, with information on the Gross Value Added from 2009 to 2014 , aims to contribute with data to the empirical evidence of the economic growth in the province of Tungurahua, marked by the development of the manufacturing sector, which explains fulfillment with the tenet of circular and cumulative causation; characterized by the interdependence between the growth of the manufacturing product and the rest of sectors of the economy in the province; at the same time it marks the difference between the economic dynamism and the other provinces of zone 3 of Ecuador.
\end{abstract}

\section{Keywords:}

Economic growth, manufactures, causation.

\section{Introducción}

La provincia de Tungurahua está reconocida a nivel nacional como una de las zonas de mayor importancia económica en el Ecuador; es tan cierta esta afirmación que en la medición de algunos indicadores macroeconómicos, como por ejemplo el Índice de Confianza del Consumidor (BCE, 2016) o del Empleo (INEC, 2015), considera a la ciudad de Ambato entre las cinco ciudades más importantes del país para la construcción de estas variables.

El desarrollo de la actividad manufacturera de la provincia de Tungurahua, ha generado una base de industrias que además de apoyar al proceso de sustitución de importaciones, se suma a la oferta exportable del Ecuador. Se pueden citar ejemplos de empresas como Plasticaucho (productora de calzado, botas de caucho, entre otros) que exporta el $40 \%$ de su producción a Perú, Colombia; o Fairis que abastece con vidrios al 95\% de la producción de electrodomésticos del país y adicionalmente exporta a México, Perú, Colombia; o Industria Metalmecánica Cepeda que produce carrocerías para buses, cuya demanda es a nivel nacional (Diario El Comercio, 2013). Por consiguiente, existe un importante aporte de la manufactura en el crecimiento económico que genera un círculo virtuoso en la provincia, mejora sus indicadores y la ubica como la provincia con mayor crecimiento de la Zona 3.

En este contexto, el presente trabajo tiene como objetivos: a) contrastar el enunciado de la primera Ley de Kaldor con la re-

Economista. Máster en Economía y Regulación de Servicios Públicos. Docente. Universidad de las Fuerzas Armadas-ESPE, Ecuador (ggcoello@espe.edu.ec). lación de causalidad entre el crecimiento del sector industrial manufacturero y el crecimiento del Valor Agregado Bruto en la provincia de Tungurahua; y b) analizar el crecimiento económico de la provincia de Tungurahua respecto de otras provincias que conforman la Zona 3 del Ecuador, y las características de los componentes sectoriales, especialmente de la manufactura, lo que determina su dinámica y su diferenciación, generando un círculo virtuoso de causación acumulativa (MYRDAL,1979).

\section{Metodología}

La investigación se efectuó a través de un estudio exploratorio, descriptivo, transversal y no experimental, utilizando fuentes bibliográficas, relacionadas con investigaciones nacionales e internacionales previas en donde se analizan las variables que determinan las diferencias del crecimiento económico entre las distintas regiones. La información estadística utilizada procede de la publicación de las Cuentas Nacionales realizadas por el Banco Central del Ecuador (BCE), específicamente de los datos correspondientes al Valor Agregado Bruto (VAB) cantonal y por sectores de la economía. El análisis se circunscribe a las cuatro provincias que conforman la Zona 3 (Tungurahua, Cotopaxi, Chimborazo y Pastaza) durante el periodo 2009-2014.

\section{Contenido}

Nicolás Kaldor, economista perteneciente a la Escuela Postkeynesiana, hizo grandes aportaciones para entender el proceso de crecimiento, dando origen a las hoy conocidas Leyes del Crecimiento de Kaldor, las cuales no fueron aceptadas por la ortodoxia económica. La primera Ley de Kaldor afirma que existe una fuerte relación de causalidad que va del crecimiento del producto manufacturero al crecimiento del PIB; con la particularidad de que el sector industrial por sí solo no es el que genera el crecimiento sino la correlación entre las dos variables, que permite que los recursos subutilizados o desempleados o de baja productividad en el sector primario o en el de servicios se trasladen a la industria, sin reducir la oferta de los demás sectores. Otro aspecto importante dentro de esta Ley es el efecto multiplicador de la manufactura, debido a que la variación del ingreso por el crecimiento de este sector incrementa significativamente la demanda de bienes y servicios de todos los sectores, genera encadenamientos productivos, promueve procesos de aprendizaje conforme crece la división del trabajo, con los consecuentes resultados de especialización y aumento de la productividad del trabajo. (SANCHEZ \& CAMPOS, 2010).

Por otro lado, Gunnar Myrlad se refirió a la causación circular acumulativa, sea de orden descendente o ascendente -según genere pobreza o promueva la acumulación- como aquel modelo que puede iniciarse por cualquier cambio importante y persistente que altere substancialmente las variables económicas: un cambio en las relaciones de intercambio, la demanda, la capacidad de ganar dinero, la inversión, la producción, etc. Además, pueden existir regiones que ofrezcan condiciones naturales propicias para que las actividades económicas se concentren en ellas. Sin embargo, cada vez es más admitido que la fuerza de atracción actual de algunos de los centros económicos más dinámicos tuvo su origen muchas veces en un accidente histórico: 
el inicio con éxito de alguna actividad específica (Martínez, 1998). Con la finalidad de comprobar el cumplimiento de la primera Ley de Kaldor y los planteamientos de Myrlad, al caso del crecimiento económico de la provincia de Tungurahua, se va analizar el desenvolvimiento del VAB de las cuatro provincias de la Zona 3 del Ecuador, conformada por Tungurahua, Chimborazo, Cotopaxi y Pastaza.

De acuerdo con los datos del Banco Central del Ecuador, en el año 2014 el Valor Agregado Bruto (VAB) del Ecuador alcanzó un total de USD 96.431.417.000,00 de los cuales el aporte de la Zona 3 fue de USD 6.500.027.250,00, equivalente al 6,7\%. EI VAB por provincias se resume en la Tabla 1, en donde se observa que la provincia de Tungurahua genera el 39\% del VAB de la Zona 3; es decir un VAB superior al de las demás provincias que conforman la Zona 3.

Tabla 1. Valor Agregado Bruto de las provincias de la Zona 3 del Ecuador

\section{Año 2014}

\begin{tabular}{|c|c|c|}
\hline Provincia & VAB (2014) en dólares & Porcentaje \\
\hline Tungurahua & 2.529 .219 .120 & $39 \%$ \\
\hline Chimborazo & 1.645 .283 .490 & $25 \%$ \\
\hline Cotopaxi & 1.569 .886 .540 & $24 \%$ \\
\hline Pastaza & 755.638 .100 & $12 \%$ \\
\hline Totales & 6.500 .027 .250 & $100 \%$ \\
\hline & \multicolumn{2}{|c|}{ Fuente: Cuentas Nacionales 2014 - Banco Central del Ecuador } \\
Elaboración: Coello, G. (2016)
\end{tabular}

En la Figura 1, se puede observar que Tungurahua aporta con un mayor $\mathrm{VAB}$ casi en todos los sectores, destacándose la manufactura como sector líder en producción en la provincia de Tungurahua, seguida de la actividad comercial, la cual se desarrolla como parte del proceso de comercialización de los productos manufacturados de la región. Las provincias en donde los sectores primarios de la economía son los que mayormente aportan al VAB (Cotopaxi con la agricultura y Pastaza con la minería) son las que menores niveles de VAB presentan.

En Ecuador, en el VAB del año 2014 se observa que el sector manufactura es el principal actor de la economía, con un total de USD 13.956.141.000 (aporta con el 14,5\% al VAB total del Ecuador), de los cuales la provincia de Tungurahua genera el 3\%, siendo la quinta provincia del Ecuador que aporta al VAB manufactura antecedida de Guayas, Pichincha, Manabí y Azuay; en noveno lugar está Chimborazo, en décimo cuarto Cotopaxi y en décimo octavo Pastaza (de un total de 24 provincias del Ecuador).

\section{Figura 1. Componentes del Valor Agregado Bruto por provincia de la Zona 3 del Ecuador Año \\ 2014}

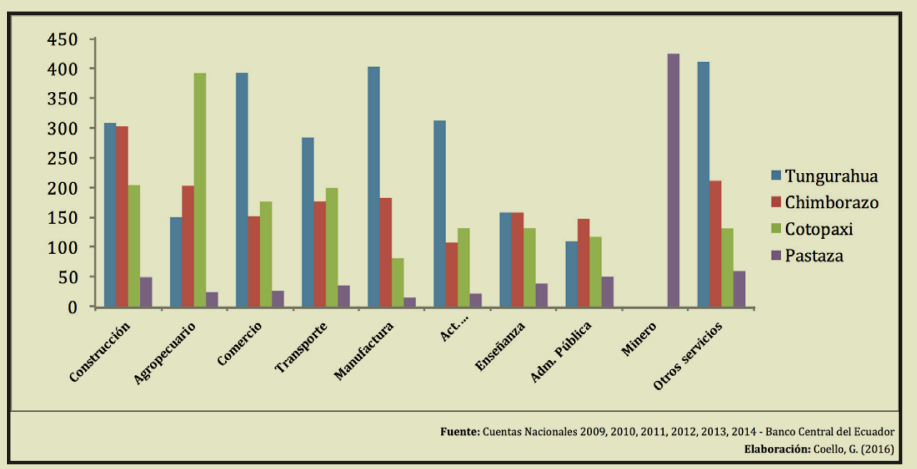

En el año 2009 la provincia de Tungurahua se encontraba en el noveno lugar en generación de producto en el sector manufactura para pasar a ocupar el puesto número cinco en el año 2014, a nivel nacional. En la tabla 2 se calculan las tasas interanuales de crecimiento del sector manufacturero de la provincia de Tungurahua, las cuales presentan variaciones; en el año 2010 se observa un alto crecimiento (66\%) el cual es producto de factores exógenos como las salvaguardias aplicadas en el año 2009, pero que fueron convertidas en oportunidades para lograr un crecimiento endógeno menos vertiginoso pero permanente en el tiempo.

\section{Tabla 2. Variación del VAB nominal y VAB Real de la Provincia de Tungurahua \\ Periodo 2009-2014}

\begin{tabular}{|c|c|c|c|c|c|c|}
\hline AÑOS & $\mathbf{2 0 0 9}$ & $\mathbf{2 0 1 0}$ & $\mathbf{2 0 1 1}$ & $\mathbf{2 0 1 2}$ & $\mathbf{2 0 1 3}$ & $\mathbf{2 0 1 4}$ \\
\hline VAB Nominal (en millones de dólares) & 138,51 & 247,71 & 313,70 & 333,27 & 357,12 & 402,19 \\
\hline VAB Real (en miles de dólares) & 120,86 & 201,14 & 241,08 & 243,95 & 253,56 & 278,96 \\
\hline Tasa de crecimiento interanual & & $66 \%$ & $20 \%$ & $1 \%$ & $4 \%$ & $10 \%$ \\
\hline & & Fuente: Cuentas Nacionales 2009, 2010, 2011, 2012, 2013, 2014 - Banco Central del Ecuador \\
Elaboración: Coello, G. (2016]
\end{tabular}

A partir de lo mencionado, como se puede mirar en la Figura 2, si comparamos la evolución en términos reales del VAB de la manufactura (sector secundario de la economía) con el VAB de la agricultura (sector primario) se observa que entre los años 2009 y 2014 , el sector primario se mantiene estable, mientras que el secundario crece de manera importante; tomando en consideración que del año 2009, el VAB real de los dos sectores parte de valores similares. Con respecto al sector terciario de la economía de Tungurahua (se compara únicamente con el comercio) se observa que ha tenido una tendencia negativa y de convergencia hacia el VAB de la manufactura; esto podría estar explicado por el hecho de que quienes realizaban actividades netamente comerciales pasaron a ser productores.

Figura 2. Tendencia de crecimiento del Valor Agregado Bruto Real de la manufactura, agricultura y comercio de la Provincia de Tungurahua Periodo 2009-2014

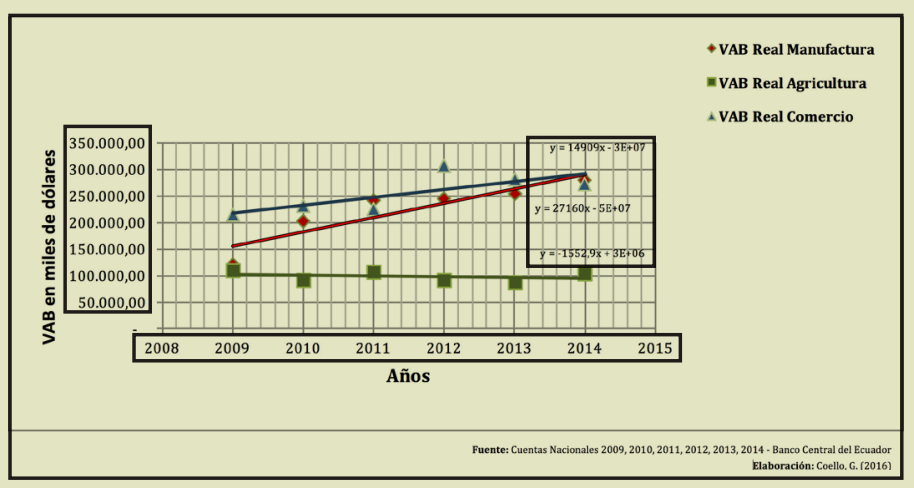

El desarrollo de la manufactura ha permitido crear una oferta exportable de la provincia de Tungurahua hacia el extranjero (USD 4.000.000,00 promedio mensual), compuesta principalmente por calzado. En la Figura 3 se presenta en resumen las exportaciones que realiza el sector manufactura de la provincia de Tungurahua (CIT, 2016). Las exportaciones se realizan básicamente a Colombia (75\%), Perú (17\%), México (5\%) y a Hong Kong, Estados Unidos y Costa Rica (3\%). 


\section{Figura 3. Exportaciones realizadas por los socios de la Cámara de Industrias de Tungurahua Periodo Octubre de 2016}

\begin{tabular}{|l}
\hline \\
\\
\\
\\
\end{tabular}

\section{Conclusiones}

En la Zona 3 del Ecuador, la provincia que genera un mayor Valor Agregado Bruto es Tungurahua. En esta provincia se observa una fuerte causalidad entre el crecimiento del producto de la manufactura y el crecimiento de la región, adicionalmente se ha dado una transferencia de recursos entre los sectores primarios (agricultura) y terciario (comercio) de la economía hacia el sector secundario (manufactura), este último con tendencia de crecimiento permanente en el periodo de análisis 2009-2014. El crecimiento de otros sectores como el de servicios, los encadenamientos productivos, el reconocimiento de productos de origen de la provincia (calzado, textiles, metalurgia), impulsa un proceso virtuoso de causación circular acumulativa de orden ascendente, que diferencia a Tungurahua de las demás provincias que conforman la Zona 3, en las cuales el sector primario de la economía es el principal generador del VAB.

El desarrollo del sector manufacturero en su correlación con el crecimiento de otros sectores de la economía tungurahuense ofrece condiciones propicias para que las actividades económicas se concentren en ella y a la vez propaguen un crecimiento económico generalizado de la provincia. De esta manera los planteamientos de los autores económicos heterodoxos Kaldor y Myrlad, sobre el crecimiento económico generado a partir del éxito de alguna actividad específica, en este caso la manufactura, son aplicables al reconocido dinamismo económico de la provincia de Tungurahua.

\section{Referencias}

- Banco Central de Ecuador. (2016). Índice de Confianza del Consumidor ICC. Recuperado en: www.bce.fin.ec

- CIT. (2016). Noti Cámara de Industrias de Tungurahua No. 040. Ambato: Cámara de Industrias de Tungurahua.

- Diario El Comercio. (11 de Noviembre de 2013). Ambato tiene industrias de exportación. pág. 1.

- Guerrero García, M., \& Pérez Martínez, Y. (2009). Procedimiento para el proceso de comercialización en cadenas de tiendas. Edición electrónica gratuita

- Instituto Nacional de Estadística y Censos. (2015). Indicadores Laborales. Recuperado en: www.ecuadorencifras.gob.ec

- Martínez, J. (1998). Procesos acumulativos y desarrollo:de Myrdal a Porter. Economía y Sociedad No. 8, 49-58

- MYRDAL, G. (1979). Teoría Económica y Regiones Subdesarrolladas. México: Fondo de Cultura Económica.

- SANCHEZ, I., \& CAMPOS, E. (2010). Industria manufacturera y crecimiento económico en la frontera norte de México. Región y Sociedad, 2.

\section{Anexo}

Artículo Implementación de una microempresa comunitaria en Tuntatacto.

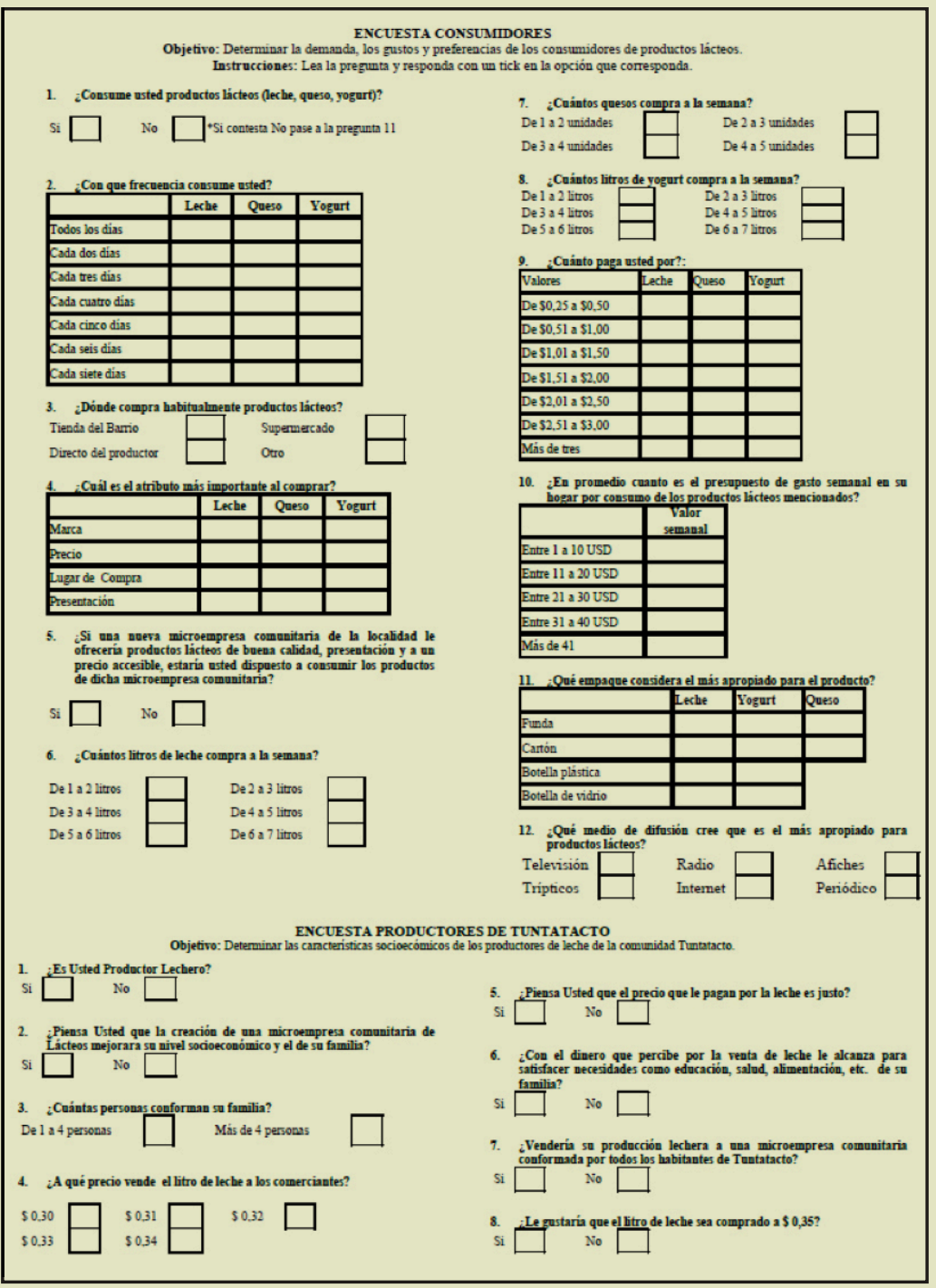

